\title{
Effectiveness of Sensors for an Arduino Microcontroller: Initial Comparisons
}

\author{
Mohd Heikal Husin \\ School of Computer \\ Sciences \\ Universiti Sains Malaysia \\ 11800 USM, Penang \\ Malaysia
}

\author{
Goh Yao Chang \\ School of Computer \\ Sciences \\ Universiti Sains Malaysia \\ 11800 USM, Penang \\ Malaysia
}

\author{
Lim Yang Kwang \\ School of Computer \\ Sciences \\ Universiti Sains Malaysia \\ 11800 USM, Penang \\ Malaysia
}

\author{
Wan Yik Mun \\ School of Computer \\ Sciences \\ Universiti Sains Malaysia, \\ 11800, USM, Penang \\ Malaysia
}

\begin{abstract}
Blindness or visual impairment has caused many difficulties for people who are suffering from such disability especially in determining obstacles and even notifying others when they are walking around at night. As such, a traditional walking cane is no longer a good aid for such users to use during their daily activities. There are several existing smart canes that are equipped with either one or two sensors that enhances the overall usage experience for the users. But, most of the existing canes implement only an obstacle detection sensor that limits the overall functionality of the cane. To overcome these weaknesses, we have proposed the development of a cane coupled with an Arduino board that includes additional sensors such as a temperature and light sensor that provides more information to the visually impaired. Due to the essential role that such sensors play for the enhancement of the proposed smart cane technology, the aim of this paper is to explore and initially identify effective sensors that is readily available for the mentioned microcontroller.
\end{abstract}

\section{General Terms}

Hardware - sensors devices and platforms, Social and professional topics - people with disabilities

\section{Keywords}

Assistive technology; microcontrollers; obstacle detection sensor; temperature sensor; light sensor

\section{INTRODUCTION}

Most people usually get their first impressions through their vision. Pawar and Mahajan [19] found that around $83 \%$ of the information that we get is based from what we see. However, people who are suffering from blindness or visual impairment are not as lucky as others. They can only retrieve the information using their sensory organs such as touch, smell and hearing. To navigate their surroundings, they need to rely on their alternative senses. Such reliance to the senses may cause some issues in their daily activities especially during navigating from a place to another place with a traditional walking cane. Their inability to map physical objects visually poses a danger as they might not know what they are walking into, eventually causing them to knock into the obstacle or even fall. Current solutions for such users would be the traditional walking cane or guide dogs, which is a rare sight in Malaysia [17]. The standard walking cane has been in use for several decades with little or no innovation [29]. Typically for new visually impaired users, the walking cane would require a lot of training and practice for the user to adapt to using the cane. Hence, the idea of a Smart White Cane has been proposed by several researchers to further extend the functionality of a normal white cane by enhancing the overall experience of the user while using it [29]. These canes are equipped with obstacle sensors that can detect objects and obstacles [10]. But, all the existing canes could only provide obstacle detection without other available information for the users. As such, we are proposing a white cane that is embedded with additional sensors such as a light sensor, an ultrasonic sensor and a temperature sensor. The sensors were selected because they could serve different functions to assist the blind users' in their day-to-day activities. There are several types of microcontroller that exists on the market such as Arduino, Raspberry, Nanode and Teensy [10]. We had selected the Arduino board due to several reasons such as low cost, accessible, ease of use, cross-platform and the availability via open source [10].

In this paper, we highlight and explore the different types of sensors that have been developed for microcontroller boards specifically an Arduino board. For section two, we highlight the background of the existing problem that led to the development of the proposed solution. This is then followed by the research objective and methodology for this research. In following sections, we then explore and compare the three existing sensors for obstacle detections, temperature as well as light sensors. Besides that, we also determine the effectiveness of the different sensors for different usage environment. Lastly, we provide our conclusions based on the comparisons of sensors. It is hoped that an effective sensor could be identified and implemented in a more efficient manner along with the traditional walking cane.

\section{BACKGROUND OF THE PROBLEM}

Nowadays, nearly $70 \%$ of visually impaired people face many issues in their daily life that has led to their dependency on others for their daily tasks [29]. They would normally use a cane that enables them to identify the path and any obstacles near to them [17]. This reliance on the cane has a huge impact to them especially in their confidence level whenever they are out on their own. Most visually impaired are often relying on a companion whenever they want to go out. After a period, some visually impaired users tend to lose their confidence or even felt ashamed as it is hard for them to be independent either at home, workplace or in public areas. Besides that, the traditional white walking cane available in the market only serves as an extension of their arm to feel the terrain or texture of the physical appearance of an obstacle or surface approximately about 1 meter ahead of them. From our research, most of the white canes do not have many sensors to help the blind people in an effective manner. In saying that, there are two existing smart cane products that can be found on the market: 1) the Smart Cane and 2) the Smart White Cane. The Smart Cane is produced by researchers from the Indian Institute of Technology Delhi in 2013 [10]. The Smart White Cane, on the other hand, is produced by another group of Indian researchers in 2014 [23]. These two products do not 
have any major differences besides the implementation of different obstacle sensors in each of the canes.

Both products are embedded with an ultrasonic sensor which is not enough to assist visually impaired people with other issues such as weather changes and dark environments. The existing number and type of sensor that the products used is too limited which could not help blind users in their daily life. Hence, this has led for us to propose our new solution that could assist the blind users in their daily life while improving the existing white cane. As part of the proposed enhancement for our Smart Enhanced White Cane solution, the identification and comparison study on the effectiveness of existing sensors would enable us to develop a more efficient product that is suited to the visually impaired.

\section{RESEARCH OBJECTIVE AND METHODOLOGY}

The main goal of this research is to study the effectiveness of different existing sensors specifically for three main usages: 1) obstacle detection, 2) temperature detection and, 3) light detection. There are three objectives in this research. The first objective is to examine the strengths and limitations of each sensor types. This is then followed by the comparison of existing sensors from different manufacturers. The comparisons would be based on the existing literature from other researchers as well as the technical specification reports from the manufacturers of each sensor. Lastly, based on the comparisons, the most effective sensors would be selected in this paper.

This research is conducted by reviewing several existing literatures by other researchers that have examined specific sensors for the main usage highlighted earlier. Besides that, the researchers also reviewed technical specification documents provided by the sensor manufacturers to compare the overall technical capabilities of the different sensors. We selected the existing literature based on experiments or comparisons that was done on obstacle detection sensors, temperature sensors and light sensors. There are nine existing literatures that have been examined for the obstacle detection sensors, five existing literatures for the temperature sensor and another 12 existing literatures for the light sensor.

\section{OBSTACLE DETECTION SENSOR}

As we know, there are several types of obstacle detection sensors that exist in the market. For this research paper, we have identified some of the frequently utilized obstacle detection sensor to identify the most effective sensor. The selected sensors are the ultrasonic sensor, infrared sensor and the laser sensor.

It is important to note that there are two types of infrared sensors which are the passive infrared sensor and the active infrared sensor. Passive infrared sensors are only able to detect an infrared beam while, the active infrared sensor can both detect and produce an infrared beam [10]. The infrared sensor that we are comparing in this section is the active infrared sensor. Although both sensors have similar working principles that detects infrared beams to detect obstacles, the sensors do act differently from each other which can be seen in the section on infrared sensors.

\subsection{Sensor Working Principles / Algorithm}

\subsubsection{Ultrasonic Sensor}

Distance $=($ Time $x$ Speed of sound $) / 2$

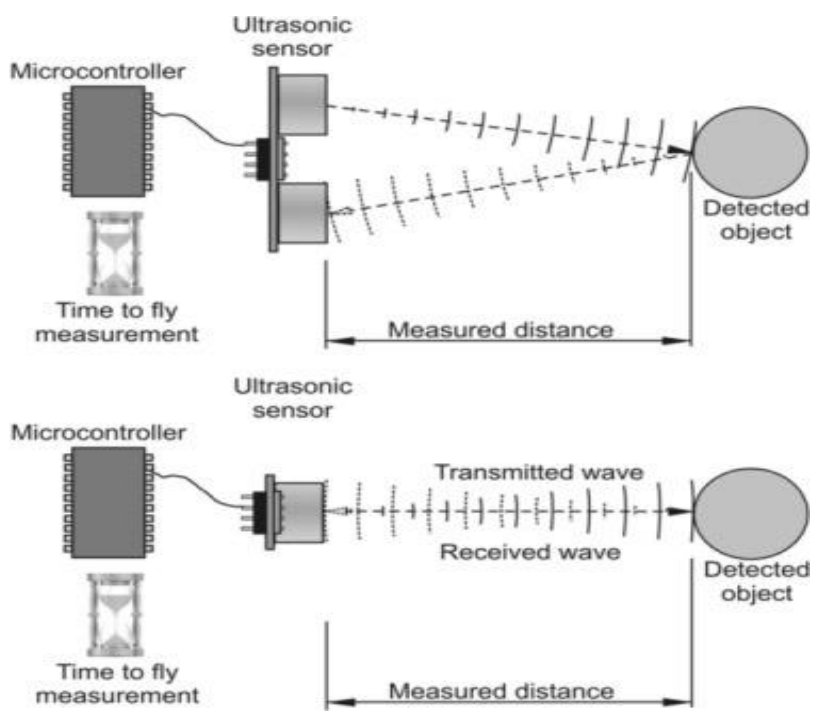

Fig 1: How ultrasonic sensor work [23]

Ultrasonic sensor is fundamentally designed for contactless distance measurement via transmitters that transmit and receive the ultrasonic sound waves. The transmitter on the sensor will transmit the sound frequency above $18 \mathrm{kHz}$ (varies between different models of sensors) through the air and at a speed of 344 meter per second (at $20^{\circ} \mathrm{C}$ ). The speed can change depending on the temperature. The receiver then receives the reflected sound from the object where it utilizes the information along with the time difference between sending and receiving the sound pulse to determine the distance of the object. The distance can be calculated by the formula below [23]. Figure 1 depicts how the ultrasonic sensor works in detail.

\subsubsection{Infrared sensor}

The second sensor that is being compared is the infrared sensor. It is a type of sensor that works similarly to the ultrasonic sensor but uses a light source to detect obstacle for contactless distance measurement. The infrared sensor also consists of two sections which are the emitter and detector. The emitter is an infrared LED and the detector is an infrared photodiode which is sensitive to the infrared light emitted by the emitter. When an object is close to the sensor, the infrared light from the LED bounces back from the object and into the photodiode. The light is then changed in intensity of light and converted to voltage. The voltage is then digitalized by using an analogue-digital converter to calculate the distance [11].

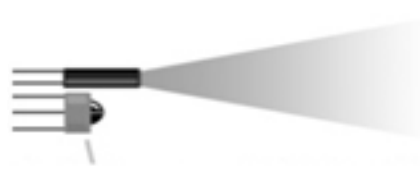

No object present - no IR light detected by sensor

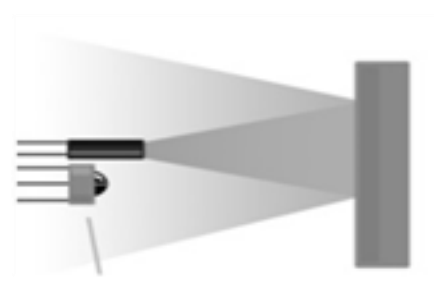

Obiect present - reflected IR light detected bv sensor

Fig 2: How infrared sensor works [11] 


\subsubsection{Laser Sensor}

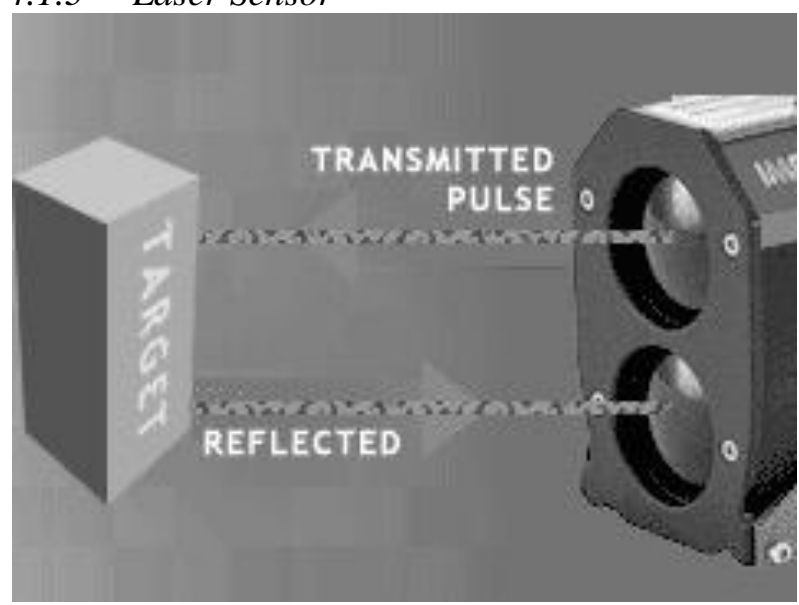

Fig 3: How laser sensor works [21]

The laser sensor is another sensor that is designed for contactless distance measurement similarly to the first two sensors. This sensor's working principle is almost similar with both the ultrasonic sensor and infrared sensor except for the laser light. The transmitter emits the laser beam and when it hits an object, the light will be reflected to the sensor. It makes use of the 'time of flight' principle which is because laser light travels at a fairly constant speed through the Earth's atmosphere. The distance can be calculated based on the formula below [21]. Figure 3 shows how the laser sensor works in further detail.

$\mathbf{c}=$ speed of light

$\mathbf{t}=$ the amount of time for the round trip between sensor and object

\subsection{Factors that Affects the Accuracy of Obstacle Sensors}

Due to the frequency and different type of sources that the different sensors emit, there are some conditions that may affect their range to transmit through the air which directly affect their overall accuracy.

\subsubsection{Ultrasonic Sensor}

As mentioned in the section earlier, the ultrasonic sensor is measured by the distance when the ultrasonic sound wave hits an object and returns to the sensor. Therefore, different conditions that affect the transverse of sound waves will also affect the accuracy of the ultrasonic sensor. Example of these conditions are as follow [4]:

- The density of the obstacle's surface (high density preferred)

- Overall noise within the air

- Environment conditions such as temperature, humidity, pressure and more.

- Angle of the sound incident upon hitting the obstacle. If the angle redirects the wave from the sensor, then the distance may not be measured.

\subsubsection{Infrared Sensor}

The infrared sensor detects an obstacle by emitting an infrared light. Similarly, as before, there are conditions that can affect the detection accuracy for the beam of infrared light. These conditions include:

- Sunlight and other infrared beams

- The obstacle's surface color (where lighter colors preferred)

- Overall lighting condition (low light preferred)

\subsubsection{Laser Sensor}

Lastly, is the laser sensor which makes use of a light source to detect obstacles. The main difference with the other sensors, is that it makes use of a high energy light source which we call as a light beam. The conditions that may affect its accuracy albeit slightly are as follow [30]:

- Overall environment conditions (eg. weather, humidity and dust)

- The lighting condition (works in most conditions but low light is preferred)

- Obstacle properties (size, reflectivity and surface)

- Angle of the incident where the beam hits the obstacle

\subsection{Comparison among the Existing Obstacle Sensor}

Table 1. Comparison among HC-SR04 [7], GP2Y0A02YK0F [22], and SF02 [18]

\begin{tabular}{|c|c|c|c|}
\hline Sensor & Ultrasonic Sensor & Infrared Sensor & Laser Sensor \\
\hline \multirow[b]{2}{*}{ Model } & HC-SR04 & GP2Y0A02YK0F & SF02 \\
\hline & & & \\
\hline Minimum Range & $2 \mathrm{~cm}$ & $20 \mathrm{~cm}$ & $0 \mathrm{~cm}$ \\
\hline Maximum Range & $400 \mathrm{~cm}$ & $150 \mathrm{~cm}$ & $4000 \mathrm{~cm}$ \\
\hline Resolution & $0.3 \mathrm{~cm}$ & $1 \mathrm{~cm}$ & 0.39 in $(1 \mathrm{~cm})$ \\
\hline Suitable Location & Indoor/Outdoor & Indoor & Indoor/Outdoor \\
\hline
\end{tabular}


Table 1 compares the three different types and model for the obstacle detection sensors which are the HC-SR04, GP2Y0A02YK0F and SF02. From Table 1, we can see that all of them have different minimum and maximum detection range. The minimum/maximum detection range determines how well the sensor could effectively detect obstacles. Anything below or above the minimum/maximum range, the sensor is unable to detect any obstacles. Therefore, the laser sensor will be a better choice in term of overall range, followed by the ultrasonic sensor and infrared sensor because it has the highest maximum range which is $4 \mathrm{~m}$ and no minimum range of obstacle detection. This would mean that it can detect both obstacles that are very near and far. The resolution is defined as the smallest changes that can be detected by the sensors. Therefore, the sensor with the smallest resolution is preferred in this case. As can be seen from Table 1, the ultrasonic sensor has the smallest resolution range among the sensor at $0.3 \mathrm{~cm}$. This allows the sensor to accurately detect small changes within the detection range.

For the infrared sensor, it is easily affected by the sunlight and other IR lights which causes it to be not suitable for outdoor use. Besides that, its range of detection is not that ideal in terms of the detection range and the accuracy is easily affected by an obstacle's surface color. From an existing research paper on the performance of ultrasonic and infrared sensors on obstacle detection, it mentioned that by using different surface colors has led to a high error percentage for the infrared sensor when used on darker surfaces. For the ultrasonic sensor, different density materials have different accuracy levels. Their experiment also highlighted that the infrared sensor has a marginally superior accuracy (around $94.7 \%$ to $99.5 \%$ ) compared to the ultrasonic sensor at an angle of $0^{\circ}$ [3]. Therefore, the infrared sensor is only suggested when there is a need for faster response time in real time tracking within a short range. We also found that there is not much researches that compare the laser sensor with other existing sensors. This may be caused by the high cost of the laser sensor to conduct such comparisons. Theoretically, the laser sensor has better performance than the ultrasonic sensor due to the high energy light beam which functions under most conditions. The laser light is also less likely to disperse like white light, which means that laser can travel much greater distance without losing its intensity. We conclude that in terms of overall effectiveness, the laser sensor has the best performance among the existing obstacle detection sensors.

\section{TEMPERATURE SENSOR}

In this section, we would be exploring the temperature sensors that could be used with the Arduino board. A temperature sensor is a device that measures the overall temperature (hot or cold) of an object. There are a few existing types of temperature sensors. They are the 1) Negative Temperature Coefficient (NTC) thermistor, 2) Resistance Temperature Detector (RTD) and, the 3) Thermocouple and Semiconductor-based sensors [12].

\subsection{Working Principle / Algorithm}

The Arduino board's analogue pins read a voltage that is expected to range between $0 \mathrm{~V}$ to $5 \mathrm{~V}$. A standard way to turn a resistance charge within a thermistor to something that the board can read (a voltage charge) is to create a voltage divider circuit. The circuit uses two resistors in a circuit with a known voltage to create a scientifically expected voltage value called Vout. The circuit is shown below in Figure 4. As the R1 (Resistor 1) value changes, the Vout also changes. R1 is the thermistor and its value would change comparative to the temperature. The Vout is then connected to the analog port on the Arduino board.

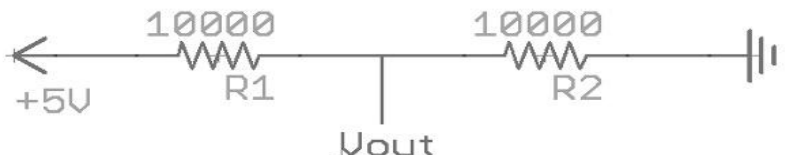

Fig 4: Voltage divider circuit schematic [16]

\subsection{Factor that Affects the Accuracy of Temperature Sensors}

Power supply voltages can affect the temperature accuracy within a temperature sensor. It is known that sensor devices with a lower regulation level of internal voltage would show greater reductions in accuracy when the power supply changes from nominal voltages. Most sensor manufacturers would include this information in their datasheet specifications, where the standard maximum values falls within the range of $\pm 0.2^{\circ} \mathrm{C} / \mathrm{V}$ to $\pm 0.3^{\circ} \mathrm{C} / \mathrm{V}$. In higher accuracy devices with $< \pm 0.5^{\circ} \mathrm{C}$ error, secondary effects usually begins to emerge that can affect the overall accuracy of the sensor. The other factors include repeatability or noise. These factors are derived from the error introduced by the analog front-end and analog-todigital converters (ADC). This can range between $\pm 0.1^{\circ} \mathrm{C}$ in lower performance products to $\pm 0.01^{\circ} \mathrm{C}$ in higher performance products. The 'Drift' or stability is also another factor. Drift refers to the electronic aging of the components in a sensor [13]. As the sensor is utilized more or matures, the accuracy is impacted. Some sensors could be adjusted when this occurs. Additional error could be introduced over time as the device ages [13].

\subsection{Comparison between the Existing Temperature Sensors}

Table 2 compares the three types of temperature sensor: 1) LM35, 2) DHT11 and the 3) DS18B20. LM35 has the biggest range of temperature measurement which is from $-55^{\circ} \mathrm{C}$ to $150^{\circ} \mathrm{C}$ while the DS18B20 has the highest accuracy of temperature measurement which is $\pm 0.5^{\circ} \mathrm{C}$ accuracy from $10^{\circ} \mathrm{C}$ to $+85^{\circ} \mathrm{C}$. Based on the comparison, it can be concluded that the DS18B20 sensor is the most effective as in terms of specification and capability it seems to fulfil most usage situations. Besides that, it supports the user configurable ability to set the resolution for the temperature detection.

\section{LIGHT SENSOR}

For this section, the existing light sensors would be highlighted and examined in detail. The light sensor is an electronic device used to detect ambient light - the overall brightness or darkness of the surrounding environment. They are often referred as photocells, photodetectors or photosensors [5]. The detected ambient light is then translated into electrical signals, mostly either as currents, voltages or even resistance. The photo transistor can convert light into either a current or voltage, whereas the photo resistor is a resistor whose resistance decreases with increasing light intensity [6]. 
Table 2. Comparison among LM35[25], DHT11[2], DS18B20[15]

\begin{tabular}{|c|c|c|c|}
\hline Model & $\begin{array}{l}/ / / \\
\text { LM35 }\end{array}$ & DHT 11 & บs I \\
\hline $\begin{array}{l}\text { Temperature } \\
\text { measured range }\end{array}$ & $-55^{\circ} \mathrm{C}$ to $150^{\circ} \mathrm{C}$ & $0-50{ }^{\circ} \mathrm{C}$ & $-55^{\circ} \mathrm{C}$ to $125^{\circ} \mathrm{C}$ \\
\hline Accuracy & $\begin{array}{l}0.5^{\circ} \mathrm{C} \text { Ensured } \\
\text { Accuracy }\left(\text { at } 25^{\circ} \mathrm{C}\right), \\
\pm 1^{\circ} \mathrm{C} \text { Accuracy from } \\
-55^{\circ} \mathrm{C} \text { to } 150^{\circ} \mathrm{C}\end{array}$ & $\pm 2^{\circ} \mathrm{C}$ & $\pm 0.5^{\circ} \mathrm{C}$ accuracy from $-10^{\circ} \mathrm{C}$ to $+85^{\circ} \mathrm{C}$ \\
\hline Output & Analog & Digital & Digital \\
\hline Mounting Type & Through hole & Through hole & Through hole \\
\hline Resolution & $10 \mathrm{mV} /{ }^{\circ} \mathrm{C}$ & 16Bit & User-configurable to $9,10,11$, or 12 bits \\
\hline Applications & Remote applications & $\begin{array}{l}\text { - HVAC } \\
\text { - Dehumidifier } \\
\text { - Testing and } \\
\text { inspection } \\
\text { equipment } \\
\text { - Consumer goods } \\
\text { - Automotive } \\
\text { - Automatic control } \\
\text { - Data loggers } \\
\text { - Weather stations } \\
\text { - Home appliances } \\
\text { - Humidity regulator } \\
\text { - Medical and other } \\
\text { humidity } \\
\text { measurement and } \\
\text { control }\end{array}$ & $\begin{array}{l}\text { - Thermostatic controls } \\
\text { - Industrial systems } \\
\text { - Consumer products } \\
\text { - Thermometers, or any thermally } \\
\text { sensitive system }\end{array}$ \\
\hline
\end{tabular}

\subsection{Working Principle / Algorithm}

The photo transistor has the same working principle as a transistor. A transistor is a semiconductor device used to amplify or switch electronic signals and electrical power [20]. The transistor limits the flow of the current depending of the applied current through different pins - collector, emitter \& base. The base controls how much current can pass through the collector through to the emitter. Figure 5 depicts the overall diagram of a transistor.

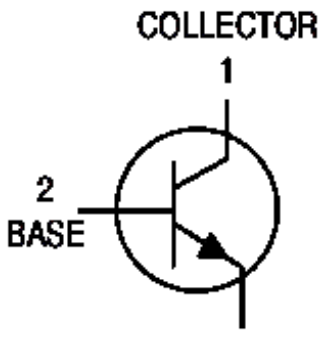

3

EMITTER

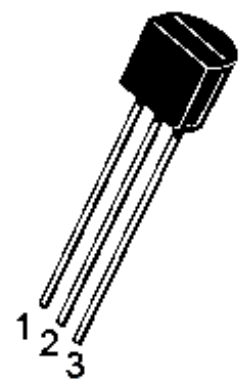

Fig 5: Circuit diagram of a transistor [20]
In a photo transistor, to determine how much current can pass through the circuit, it relies on the exposure of the light. When light falls on the junction on the base, reverse current flows in proportional to the luminance [1]. The more the light falls onto the photo transistor, the lesser the reverse current flows and hence, a larger amount of current can flow through it. Figure 6 shows the circuit diagram of a photo transistor.

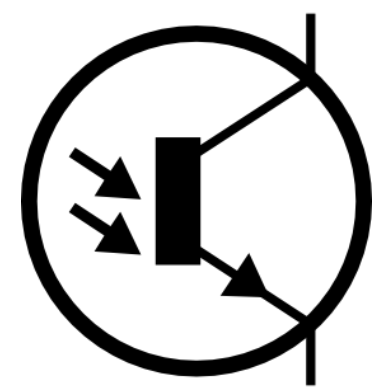

Fig.6: Circuit diagram of a photo transistor [1]

A photo resistor on the other hand, operates similarly to a photo transistor. However, it changes the resistance based on the amount of light that falls upon it. In other words, it increases the resistance as lesser light is detected by the resistor. Figure 7 shows the circuit diagram of a photo resistor.
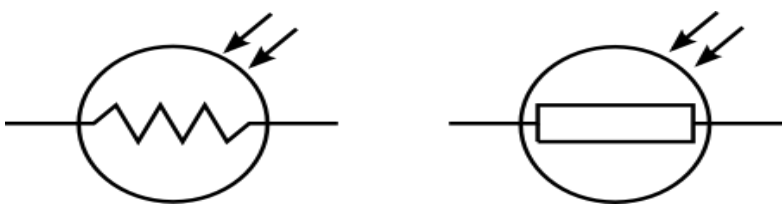

Fig 7: Circuit diagram of photo resistor [1] 


\subsection{Factors that Affects the Accuracy of Light Sensors}

The accuracy of a light sensor depends on the level of available light that hits the sensor. Photocells are more sensitive to red and green light as compared to blue light [8]. This is due to a longer wavelength in red light $(650 \mathrm{~nm})$ and green light $(510 \mathrm{~nm})$. The blue light has a wavelength of approximately $470 \mathrm{~nm}$. Besides that, red light is nearer to the infrared light in the light spectrum. This explains why light sensors are more sensitive in response to incandescent bulb rather than fluorescent bulb [9]. Dark resistance is the resistance of photocell under zero illumination lighting conditions. It defines the maximum "leakage current" that can be expected when a given voltage is applied across the photocell [26]. Each photocell has its own resistance against temperature characteristics. An increase in temperature will result in a decrease in the resistivity in the semiconductor [14]. The speed of response is the rise and decay response time in which the photocell responds to a change of light intensity of the surroundings. A higher response time will result in a poor accuracy of the light reading.

\subsection{Comparison between the Existing Light Sensors}

Table 3 compares three different photocells - PGM5506, LM393 and TEMT6000. From their working ambient temperature, TEMT6000 has a bigger range from $-40{ }^{\circ} \mathrm{C}$ to 85 ${ }^{\circ} \mathrm{C}$. It can work in both hot and below freeze temperatures. Judging by their spectral peak, the TEMT6000 can read higher spectral value, indicating that lights with higher wavelength can be identified. Based on their photo resistance, TEMT6000 has a bigger range from $3.8 \mathrm{~K} \Omega$ to $17.1 \mathrm{~K} \Omega$. This means that TEMT6000 sensor would have a more accurate reading for the light that falls on the sensor as it has a bigger range. In terms of response time, the TEMT6000 has a shorter time of $15 \mathrm{~ms}$. Hence, it will be able to generate a more accurate output based on that time the reading was taken.

Table 3. Comparison among PGM5506 [27], LM393 [24] and TEMT6000 [28]

\begin{tabular}{|c|c|c|c|}
\hline Model & PGM5506 & Pe & TEMT'6000 \\
\hline $\begin{array}{l}\text { Photodetector } \\
\text { Type }\end{array}$ & Photo resistor & $\begin{array}{l}\text { Photo } \\
\text { resistor }\end{array}$ & $\begin{array}{l}\text { Photo } \\
\text { transistor }\end{array}$ \\
\hline $\begin{array}{l}\text { Max Voltage } \\
\text { (VDC) }\end{array}$ & 100 & 36 & 6 \\
\hline $\begin{array}{c}\text { Ambient } \\
\text { Temperature } \\
\left({ }^{\circ} \mathrm{C}\right)\end{array}$ & $-30-+70$ & $0-+70$ & $-40-+85$ \\
\hline $\begin{array}{l}\text { Spectral Peak } \\
\quad(\mathrm{nm})\end{array}$ & 540 & 540 & 570 \\
\hline $\begin{array}{c}\text { Photo } \\
\text { Resistance at } \\
101 \mathrm{~K}(\mathrm{~K} \Omega)\end{array}$ & $2-6$ & $8-20$ & $3.8-17.1$ \\
\hline $\begin{array}{l}\text { Dark Resistance } \\
(\mathrm{M} \Omega) / \mathrm{min}\end{array}$ & 0.15 & 0.1 & 0.1 \\
\hline
\end{tabular}

\begin{tabular}{|c|c|c|c|}
\hline $\begin{array}{c}\text { Rise Response } \\
\text { Time (ms) }\end{array}$ & 30 & 20 & 15 \\
\hline Size (mm) & $\begin{array}{c}5.0 \\
\text { (diameter) }\end{array}$ & $22 \times 12$ & $10 \times 10$ \\
\hline $\begin{array}{c}\text { Gross Weight } \\
\text { (g) }\end{array}$ & 0.23 & 4.5 & 0.3 \\
\hline Price (USD) & 0.27 & 2.68 & 4.95 \\
\hline
\end{tabular}

\section{CONCLUSION}

From the comparison of sensors in the previous sections, we have identified that these three sensors provide the highest effectiveness in their individual category which are the 1) laser sensor SF-02 for obstacle detection, 2) the DS18B20 sensor for temperature detection and, 3) the TEMT6000 sensor for light detection. The effectiveness of the specific sensors is compared based on the datasheet provided by their manufacturers as well as existing comparison conducted by other researchers. Such comparisons may vary from different researchers or environments. As such, additional tests as well as controlled experiments based on the factors that may affect their individual accuracy would have to be carried out in relevant future works.

The results from such tests would enable us to accurately measure the significance of these factors in affecting the accuracy of the respective sensors. It is hoped that more effective sensors could be identified in enabling the visually impaired to lead a more productive and easier life despite of their disability.

\section{REFERENCES}

[1] Agarwal, T. (2015) Basics of Phototransistors - Types, Features and Applications [Online]. Available: https://www.elprocus.com/phototransistor-basics-andadvantages/.

[2] Aosong Electronics (2010) Temperature and humidity module DHT11 Product Manual [Online]. Available: https://akizukidenshi.com/download/ds/aosong/DHT11.p df.

[3] Baharuddin M., Aladin Z., Rezaul K. B. (2013) Ultrasonic and Infrared Sensor Performance in a Wireless Obstacle Detection System. in Proceedings of the $20131^{\text {st }}$ International Conference on Artificial Intelligence, Modelling and Simulation (AIMS), 3 - 5 Dec 2013, Washington DC., 487-492.

[4] Calin, D. (2013) Types of Sensors for Target Detection and Tracking [Online]. Available: https://www.intorobotics.com/types-sensors-targetdetection-tracking/.

[5] Carnegie Mellon Robotics Academy (2016) Light Sensor - What is a Light Sensor? [Online]. Available: http://www.education.rec.ri.cmu.edu/content/electronics/ boe/light_sensor/1.html.

[6] Electronics Tutorials (2016) Light Sensor [Online]. http://www.electronics-tutorials.ws/io/io_4.html.

[7] ElecFreaks (2014) HC-SR04 User Guide [Online]. Available: http://www.elecfreaks.com/wiki/index.php? title=HC-SR04_User_Guide.

[8] Hamblen, J. (2015) Using a Photocell or Phototransistor to determine lighting levels $\mid$ mbed [Online]. Available: 
https://developer.mbed.org/users/4180_1/notebook/using -a-photocell-to-determine-light-levels/.

[9] Harmon, D., Electronic Design (2015) Why Does Accuracy Matter So Much with an Ambient Light Sensor? [Online]. Available: http://electronicdesign.com/optical/why-does-accuracymatter-so-much-ambient-light-sensor.

[10] Indian Institute of Technology Delhi. (2012). Development of Smart Cane [Online]. Available: http://alumni.media.mit.edu/ djain/media/Smartcane/Sm artcane.pdf.

[11] Kelemen, M., Virgala, I., Kelemenová, T., Miková, L, Frankovský, P., Lipták, T., Lörinc, M. (2015) Distance Measurement via Using of Ultrasonic Sensor. J. of Automation and Control, 3, 3, 71-74.

[12] Kirchner, S. (2015) 4 Most Common Types of Temperature Sensor [Online]. Available: http://www.ametherm.com/blog/thermistors/temperaturesensor-types/.

[13] Lethawicker. (2015) Semiconductor Temperature Sensor Accuracy [Online]. Available: http://community.silabs.com/t5/Official-Blog-ofSiliconLabs/Semiconductor-Temperature-SensorAccuracy/ba-p/139015.

[14] Levinson, J., Shepherd, F.R., Scanlon, P.J., Westwood, D., Este, G., Rider, M. (2016) Conductivity behavior in polycrystalline semiconductor thin film transistors. $J$. of Applied Physics, 53, 1193.

[15] Maxim Integrated (2015) DS18B20 Programmable Resolution 1-Wire Digital Thermometer [Online]. Available: http://datasheets.maximintegrated.com/en/ds/ DS18B20.pdf.

[16] Miller, B. (2013) How to Read Temperatures with Arduino [Online]. Available: https://computers.tutsplus.com/tutorials/how-to-readtemperatures-with-arduino--mac-53714.

[17] Mothiravally, V., Ang, S., Baloch, G. M., Kulampallil, T. T., Geetha, S. (2014) Attitude and Perception of Visually Impaired Travellers: A Case of Klang Valley, Malaysia. in $5^{\text {th }}$ Asia-Euro Conference 2014 in Tourism, Hospitality and Gastronomy, 144, 366-377.

[18] Parallax Inc (2016) SF02 Laser Rangefinder [Online]. Available: https://www.parallax.com/product/28043.
[19] Pawar, A.R., Mahajan, M.M. (2015) Multitasking Stick for Indicating Safe Path to Visually Disable People. IOSR J. of Electron. and Communication Eng.(IOSR$J E C E), 10,3,7-11$. May -Jun 2015.

[20] Riordan, M., Hoddeson, L., Herring, C., "The invention of the transistor", Reviews of Modern Physics, vol. 71, no. 2,1999 .

[21] Seubert, C. (2016). How do Laser Distance Meters Works [Online]. Available: http://www.ehow.com/ about_6332366_do-laser-distance-meters-work_.html.

[22] Sharp Electronics (2006) Sharp GP2YOAO2YKOF [Online]. Available: http://www.sharpsma.com/ webfm_send/1487.

[23] Sheth, R., Rajandekar, S., Laddha, S., Chauhari, R. (2014). Smart White Cane - An Elegant and Economic Walking Aid [Online]. Available http://www.slideshare.net/ajer123/1031084089.

[24] Texas Instruments (2014) LMx93-N, LM2903-N LowPower, Low-Offset Voltage, Dual Comparators SNOSBJ6F datasheet October 1999, Revised December 2014 [Online]. Available: http://www.ti.com/lit/ds/symlink/lm393-n.pdf.

[25] Texas Instruments (2016) LM35 Precision Centigrade Temperature Sensors [Online]. Available: http://www.ti.com/lit/ds/symlink/lm35.pdf.

[26] Thongpron, J., Kirtikara, K. and Jivacate, C. (2006) A method for the determination of dynamic resistance of photovoltaic modules under illumination. Solar Energy Materials and Solar Cells, 90, 18-19, 3078-3084.

[27] Token Electronics (2016) CDS Light-Dependent Photoresistors [Online]. Available: http://token.com.tw/pdf/resistor/cds-resistor-pgm.pdf.

[28] Vishay Semiconductors (2011) Ambient Light Sensor TEMT6000X01 Document Number 81579 [Online] http://www.vishay.com/docs/81579/temt6000.pdf.

[29] Vishwa, B., Chary, R., Kumar, B.S. (2014) Rescue System for Visually Impaired Blind Persons. Int. J. of Eng. Trends and Technology (IJETT), 16, 4, Oct 2014.

[30] Zant, C (2013) How Do Rangefinders Work [Online]. Available: http://precisionrifleblog.com/2013/10/29/howdo-rangefinders-work/. 